\title{
Associação forética dos ácaros Myialges anchora Sergent \& Trouessart (Acaridida, Epidermoptidae) e Ornithocheyletia hallae Smiley (Actinedida, Cheyletiellidae) com Pseudolynchia canariensis (Macquart) (Diptera, Hippoboscidae)
}

\author{
Michel P. Valim ${ }^{1,2} \&$ Gilberto S. Gazêta ${ }^{1,2}$
}

${ }^{1}$ Laboratório de Ixodides, Departamento de Entomologia, Instituto Oswaldo Cruz, Fiocruz, Av. Brasil 4365, 21.045-900 Manguinhos, Rio de Janeiro-RJ, Brasil. mpvalim@ hotmail.com

${ }^{2}$ Bolsista do CNPq - Brasil

\begin{abstract}
Phoretic association of the mites Myialges anchora Sergent \& Trouessart (Acaridida, Epidermoptidae) and Ornithocheyletia hallae Smiley (Actinedida, Cheyletiellidae) with Pseudolynchia canariensis (Macquart) (Diptera, Hippoboscidae). Twenty three louse-flies, from a total of 45 specimens of Pseudolynchia canariensis examined, were found parasitised by mites of the species Myialges anchora and Ornithocheyletia hallae. These mites are recorded for the first time for the State of Rio de Janeiro, in Brazil. Data on levels of infestation, as well as on the topographical distribution of the mites on the body of the louse-flies are briefly discussed.
\end{abstract}

KEYWORDS. Dermicoles mites; louse flies; Phoresy; Brazil.

RESUMO. Associação forética dos ácaros Myialges anchora Sergent \& Trouessart (Acaridida, Epidermoptidae) e Ornithocheyletia hallae Smiley (Actinedida, Cheyletiellidae) com Pseudolynchia canariensis (Macquart) (Diptera, Hippoboscidae). A partir de 45 espécimes de Pseudolynchia canariensis examinados, foram encontrados 23 deles hiperparasitados por ácaros. É registrada a ocorrência de Myialges anchora e Ornithocheyletia hallae no estado do Rio de Janeiro, Brasil. Dados sobre o grau de infestação desses hipoboscídeos, bem como a distribuição topográfica dos ácaros sobre o corpo desses insetos são brevemente discutidos.

PALAVRAS-CHAVE. Ácaros dermícolas; foresia; pupíparos; Brasil.

Dentre as diversas famílias de ácaros que parasitam aves, os pertencentes a Epidermoptidae e Cheyletiellidae destacamse por determinar processo descamativo na base das penas, semelhante a uma sarna, porém sem penetrar profundamente na pele, permanecendo apenas em sua camada queratinizada, causando uma dermatite não pruriginosa. Entretanto, estes ácaros têm sido associados à patologia e mortalidade de filhotes, sobretudo pela irritação causada pelos ganchos presentes no primeiro par de pernas de algumas espécies do gênero Myialges Trouessart, 1906 (Philips \& Fain 1991), estando associados à severa inflamação granulomatosa, sugerindo dermatite que pode ser causa significativa de morbidade ou, até mesmo, mortalidade para aves (Gilardi et al. 2001). A infestação de um novo hospedeiro ocorre principalmente quando a fêmea fixa-se em um díptero, especialmente hipoboscídeo, onde pode fazer a postura de ovos. As formas imaturas, originadas após eclosão das larvas, são transferidas para uma nova ave hospedeira quando o inseto passa de um animal para o outro (Krantz 1978; Madden \& Harmon 1998).

No Brasil, Myialges anchora Sergent \& Trouessart, 1907 (Acaridida, Epidermoptidae) foi relatada em Pseudolynchia canariensis (Macquart, 1840) (Diptera, Hippoboscidae) por Figueredo \& Barbosa (1944) no estado de Pernambuco. Outro relato desta associação no Brasil foi feito por Feres \& Flechtmann (1991) que encontraram M. (Promyialges) lophortyx (Fumann \& Tharshis, 1953) e Ornithocheyletia hallae Smiley, 1970 (Actinedida, Cheyletiellidae) em $P$. canariensis no estado de São Paulo.

A partir de 45 espécimes de $P$. canariensis coletados em pombos domésticos, Columba livia Gmelin (Aves, Columbiformes), provenientes da região metropolitana do Rio de Janeiro, estado do Rio de Janeiro, observou-se a presença de ácaros fixados em diferentes segmentos do corpo dos hipoboscídeos. No Laboratório de Ixodides do Instituto Oswaldo Cruz (FIOCRUZ) os ácaros foram removidos com o auxílio de estilete de ponta fina e montados entre lâmina e lamínula em meio de Hoyer (Flechtmann 1990). Após montagem, os ácaros foram identificados com auxílio do trabalho de Fain (1965) e das chaves dicotômicas de Gaud \& Atyeo (1996) e Furmann \& Tharshis (1953) paraEpidermoptidae e de Smiley (1970) para a identificação de Cheyletiellidae.

Foram calculados a prevalência, o índice de abundância parasitária (Ab), a intensidade média de parasitismo (IMP) seguindo a proposição de Bush et al. (1997) e o coeficiente de dominância (CD) dos ácaros, segundo Serra-Freire (2002).

As regiões dorsais e ventrais da cabeça, tórax e abdômen, foram observadas em busca de ácaros e seus ovos, as localizações 
nas diferentes regiões foram identificadas e os ácaros contados.

Dos 45 hipoboscídeos examinados 23 estavam infestados, sendo removidos 77 ácaros de diferentes partes do corpo. Destes, 40 eram fêmeas de $M$. anchora e estavam fixadas em várias partes do corpo do díptero; e 37 fêmeas de $O$. hallae, não fixadas, encontradas entre o metatórax e o primeiro tergito abdominal. A distribuição de $M$. anchora no corpo dos hipoboscídeos, juntamente com o número de ovos encontrados está apresentada na Tabela I.

A prevalência encontrada para $M$. anchora foi de $37,78 \%$ (17/45), a Ab e a IMP, de 0,89 e 2,35, respectivamente. Para $O$. hallae a prevalência foi de 20\% (9/45); a Ab e IMP, de 0,82 e 4,11 , respectivamente. Considerando as duas espécies, a prevalência foi de 51,11\% (23/45), a Ab de 1,71 e a IMP igual a 3,35. O índice de coeficiente de dominância foi de $52 \%$ para $M$. anchora e de $48 \%$ para $O$. hallae. Das nove moscas que apresentavam ácaros queiletielídeos, em três (33,3\%) foram encontrados ácaros epidermoptídeos.

Não foram encontrados ovos no local de presença de $O$. hallae. No processo de foresia, relatado por Feres \& Flechtmann (1991) para esta espécie, os ácaros foram encontrados fracamente aderidos ao corpo do forético e sem presença de ovos. Embora este seja o segundo relato de foresia por esta espécie, é possível que os mesmos não adotem o hábito de oviposição nos hospedeiros de transporte.

Bequaert (1955:354) encontrou 2,2\% de Icosta hirsuta (Ferris, 1927) parasitadas por M. anchora e M. lophortyx, valor muito inferior ao encontrado neste trabalho. Entretanto, Bequaert (1953) relata que 54,8\% de $P$. canariensis carreavam ácaros no Congo, sendo esta prevalência mais equiparável ao valor encontrado, a exemplo de estudo realizado na Argentina (Bequaert 1953), onde a prevalência por sexo de hipoboscídeos infestados foi de $18 \%$ e $12,5 \%$ para fêmeas e machos, respectivamente. Jovani et al. (2001) encontraram 49,5\% para Epidermoptidae e 26,7\% para Cheyletiellidae, o que, associado à prevalência ora obtida, mostra a nítida dominância de Epidermoptidae, fato comprovado pelo coeficiente de dominância de 54\% e 46\%, nas respectivas famílias.

Sergent \& Trouessart (1907) apud Bequaert (1953: 145), relataram $M$. anchora, cercados por cachos de ovos nas regiões da cabeça, tórax e abdome dos hipoboscídeos. Os mesmos autores relatam que a maioria dos ácaros foram encontrados no abdome, porém 29,4\% (5/17) das moscas infestadas apresentavam fêmeas e/ou ovos na membrana da base da peça bucal e apenas uma mosca $(5,9 \%)$ apresentava uma fêmea aderida à área basal de uma das asas. No presente estudo também foram encontrados ácaros nessas localizações, sendo 7,5\% (3/40) na base da peça bucal e 2,5\% (1/40) nas asas. Encontrou-se, ainda, ácaros acima das peças bucais e no trocanter do segundo par de pernas, ambos com 2,5\% (1/ 40) de freqüência.

Com base nos resultados encontrados, os ácaros epidermoptídeos utilizam hipoboscídeos como forma de dispersão, ocorrendo preferencialmente na região dorsal do abdome para fixação.
Tabela I. Distribuição de Myalges anchora e seus ovos (entre parênteses) nas diferentes regiões do corpo de Pseudolynchia canariensis da região metropolitana do Rio de Janeiro-RJ, Brasil.

\begin{tabular}{llll}
\hline Regiões do corpo & Face dorsal & Face ventral & Total \\
\hline Cabeça & $01(31)$ & $02(00)$ & $03(31)$ \\
Tórax & $02(74)$ & $02(30)$ & $04(104)$ \\
Abdomen & $24(184)$ & $09(05)$ & $33(189)$ \\
Total & $27(289)$ & $13(35)$ & $40(324)$ \\
\hline
\end{tabular}

Agradecimentos. Ao Dr. Reinaldo J. F. Feres da Universidade Estadual Paulista (UNESP), São José do Rio Preto-SP, Brasil; ao Dr. Carlos H. W. Flechtmann da Universidade de São Paulo, Escola Superior de Agricultura Luiz de Queiroz (USP-ESALQ), Piracicaba-SP, Brasil pela leitura crítica e sugestões na versão original; e aos revisores da Revista Brasileira de Entomologia pelas valiosas críticas e sugestões.

\section{REFERÊNCIAS}

Bequaert, J. G. 1953. The Hippoboscidae or louse-flies (Diptera) of mammals and birds. Part I. Structure, Physiology and Natural History. Entomologica Americana 32: 233-416.

Bequaert, J. G. 1955. The Hippoboscidae or louse-flies (Diptera) of mammals and birds. Part II. Taxonomy, Evolution and Revision of American Genera and Species. Entomologica Americana 35: 233416.

Bush, A. O.; K. D. Lafferty; J. M. Lotz \& A. W. Shostak. 1997. Parasitology meets ecology on its own terms: Margolis et al. revisited. Journal of Parasitology 83: 575-583.

Fain, A. 1965. A review of the family Epidermoptidae Trouessart parasitic on the skin of birds. Koninklijke Vlaamse Academie Voor Weten-Schappen, Letteren en Schone Kunsten van België 84: 1-176 (Part I), 1-144 (Part II).

Feres, R. J. F. \& C. H. W. Flechtmann. 1991. Ocorrência de ácaros "Parasitos-Foréticos" (Acari: Epidermoptidae, Cheyletiellidae) sobre moscas hipoboscídeas de pombo, em São José do Rio Preto, SP. Naturalia 16: 155-160.

Figueredo, A. \& F. A. S. Barbosa. 1944. A propósito do hiperparasitismo de "Pseudolynchia maura" (Diptera: Hippoboscidae). Anais da Sociedade Biológica de Pernambuco 5: 15-18.

Flechtmann, C. H. W. 1990. Ácaros de Importância MédicoVeterinária. $3^{a}$ ed., Editora Nobel, São Paulo, 192 p.

Furmann, D. P. \& I. B. Tharshis. 1953. Mites of the genera Myialges and Microlichus (Acarina: Epidermoptidae) from avian and insect hosts. Journal of Parasitology 39: 70-78.

Gaud, J. \& W. T. Atyeo 1996. Feather mites ofthe World (Part 1). Annales Musée Royal de L’Afrique Centrale, Sciences Zoologiques 277: 1-193.

Gilardi, K. V.; J. D. Gilardi; A. Frank; M. L. Goff \& W. M. Boyle. 2001. Epidermoptid mange in Laysan albatross fledglings in Hawai. Journal of Wildlife Disease 37: 185-188.

Jovani, R.; J. L. Tella; D. Sol, \& D. Ventura. 2001. Are hippoboscid flies a major mode of transmission of feather mites? Journal of Parasitology 87: 1187-1189.

Krantz, G. W. 1978. A Manual of Acarology. 2nd., Corvallis, Oregon State University Book Store, 508 p.

Madden, D. \& W. M. Harmon. 1998. First record and morphology of Myialges caulotoon (Acari: Epidermoptidae) from Galapagos Hosts. Journal of Parasitology 84: 186-189.

Philips, J. R. \& A. Fain. 1991. Acarine symbionts of louseflies (Diptera: Hippoboscidae). Acarologia 32: 377-384.

Serra-Freire, N. M. 2002. Planejamento e Análise de Pesquisas Parasitológicas. Niterói, Editora da Universidade Federal Fluminense, 199 p.

Smiley, R. L. 1970. A review of the family Cheyletiellidae (Acarina). Annals of the Entomological Society of America 63: 10561078 . 